\title{
Direct Oxytosylation of 8-Amidoquinolines by Koser's Reagent: An Efficient Strategy for 5-Substituted 8-Amidoquinolines
}

\author{
Zubeda Beguma \\ G. Bhavani ${ }^{a}$ \\ B. Sridhar ${ }^{b}$ \\ Basireddy V. Subba Reddy*a \\ ${ }^{a}$ Center for Semiochemicals, CSIR-Indian Institute of Chemical \\ Technology, Hyderabad 500007, India \\ bvsreddyiict@gmail.com \\ b Laboratory of X-ray Crystallography, CSIR-Indian Institute of \\ Chemical Technology, Hyderabad 500007, India
}
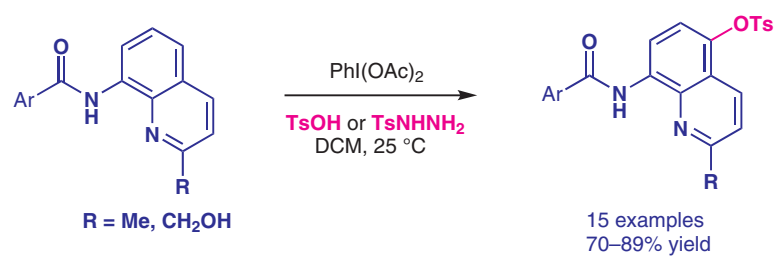

sphere. ${ }^{8}$ The Koser's reagent was prepared from $p$-toluenesulfonic acid or $p$-toluenesulfonyl hydrazide using readily available (diacetoxyiodo)benzene (Scheme 1). ${ }^{9}$

Accepted after revision: 20.06.2018

Published online: 07.08 .2018

DOI: 10.1055/s-0037-1610212; Art ID: ss-2018-t0022-op

Abstract A metal-free remote oxytosylation of 8-amidoquinolines has been achieved using Koser's reagent to produce 5-tosyloxy-8-amidoquinolines in good yields. This method is compatible with various functional groups present on the aromatic ring.

Key words Koser's reagent, $\mathrm{C}-\mathrm{H}$ activation, 8-amidoquinolines, oxytosylation, hypervalent iodine reagents

Introduction of oxygen functionality on aromatic and heteroaromatic ring is a challenging task in organic synthesis. ${ }^{1}$ Recently, a few strategies have been developed for the oxytosylation of aromatic systems through a $\mathrm{C}-\mathrm{H}$ bond oxidation. ${ }^{2}$ In recent years, 8-amidoquinolines have successfully been employed for the aromatic $\mathrm{C}-\mathrm{H}$ bond functionalization. ${ }^{3}$ On the other hand, a remote functionalization of 8 amidoquinolines have been reported with diversified reagents to generate 5-substituted 8-amidoquinolines through a C-H activation. ${ }^{4}$ In recent years, Koser's reagent 2 has gained importance as a versatile and effective source for oxytosylation of organic compounds. ${ }^{5}$ Recently, Shen et al. ${ }^{6}$ reported the iodobenzene-catalyzed synthesis of aryl sulfonate esters by the remote functionalization of aminoquinolines at room temperature using peracetic acid as oxidant. However, there have been no reports on oxytosylation of 8-amidoquinolines at C-5 position using Koser's reagent.

Following our interest on $\mathrm{C}-\mathrm{H}$ functionalization, ${ }^{7}$ we herein report an efficient strategy for the synthesis of 5-tosyloxy-8-amidoquinolines using Koser's reagent, which is generated in situ from $\mathrm{PhI}(\mathrm{OAc})_{2}$ and $p$-toluenesulfonic acid $(p$-TSA). The required precursors were prepared from 8aminoquinoline and the corresponding carboxylic acid using EDCI and DMAP in $\mathrm{CH}_{2} \mathrm{Cl}_{2}$ at $0{ }^{\circ} \mathrm{C}$ under nitrogen atmo-
To optimize the reaction conditions, the oxytosylation of $N$-(quinolin-8-yl)benzamide (1a) with $p$-toluenesulfonic acid or $p$-toluenesulfonyl hydrazide was attempted in the presence of $20 \mathrm{~mol} \% \mathrm{PhI}(\mathrm{OAc})_{2}$ in dichloromethane (Scheme 2).
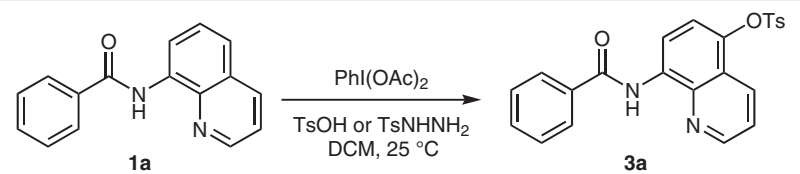

Scheme 2 Oxytosylation of $N$-(quinolin-8-yl)benzamide (1a)

The product 3a was isolated in low yield (20\%) under these conditions (Table 1, entry 1 ). Therefore, the above reaction was carried out using a stoichiometric amount of $\mathrm{PhI}(\mathrm{OAc})_{2}$. Interestingly, the product 3a was obtained in excellent yield under the above conditions (entry 2). However, no reaction was observed in the absence of $\mathrm{PhI}(\mathrm{OAc})_{2}$ (entry 3 ). The product 3a was obtained only in $40 \%$ yield when the reaction was performed in acetonitrile (entry 4 ).

These initial findings encouraged us to study the scope of this methodology. This method is compatible with different 8-amidoquinolines bearing chloro-, bromo-, fluoro-, methyl-, and trifluoromethyl substituents on the aromatic

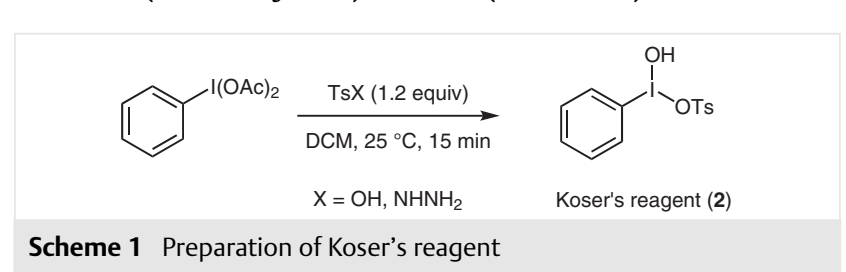


Table 1 Optimization of Reaction Conditions in the Formation of $3 a^{a}$

\begin{tabular}{|c|c|c|c|c|c|}
\hline Entry & $\mathrm{Phl}(\mathrm{OAc})_{2}$ (equiv) & $p$-TSA (equiv) & Solvent & Time (h) & Yield (\%) \\
\hline 1 & 0.2 & 1.2 & $\mathrm{CH}_{2} \mathrm{Cl}_{2}$ & 1.0 & 20 \\
\hline 2 & 1.05 & 1.2 & $\mathrm{CH}_{2} \mathrm{Cl}_{2}$ & 12 & 86 \\
\hline 3 & 0 & 1.2 & $\mathrm{CH}_{2} \mathrm{Cl}_{2}$ & 6 & 0 \\
\hline 4 & 1.0 & 1.2 & $\mathrm{MeCN}$ & 12 & 40 \\
\hline
\end{tabular}

a Reaction was performed in $1.5 \mathrm{mmol}$ scale at $25^{\circ} \mathrm{C}$.

ring of the carboxylic acid moiety (Scheme 3 ). The substituent present on the aryl group of 8-amidoquinolines had shown significant effect on the conversion. The presence of halide or methyl group on the aryl ring gave the product in good yield. The reaction was quite successful not only with aromatic but also with heteroaromatic substrates (3k). However, the reaction was unsuccessful with amides derived from 8-aminoquinoline and carboxylic acids like 4-nitrobenzoic acid and acetic acid (3p and 3q). Similarly, the amide derived from aniline and benzoic acid also failed to give the desired product (3r). Furthermore, no desired product was obtained either with 8-aminoquinoline or with $\mathrm{NBoc}$, and $\mathrm{NCbz}$ derivative of 8-aminoquinoline. All the products were thoroughly characterized by NMR, IR, and mass spectrometry. The reaction is highly selective and no oxytosylation was observed on the aryl ring of the carboxylic acid moiety (Scheme 3 ). This method was successfully applied for a gram scale $(1.2 \mathrm{~g})$ synthesis of product 3d.

Finally, the structure of 3a was confirmed unambiguously by a single crystal X-ray structural analysis (Figure 1). ${ }^{10}$

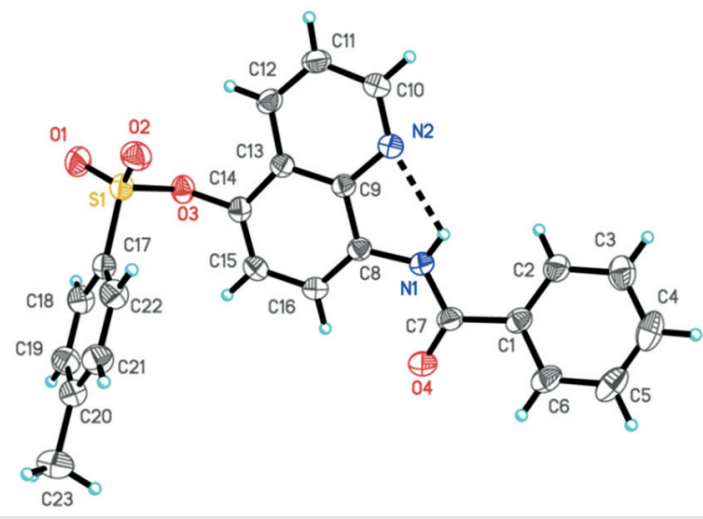

Figure 1 ORTEP diagram of $3 a$

In addition, a differential deprotection of both tosyl and amide groups has been achieved by merely changing the reaction conditions (Scheme 4). Deprotection of the amide functionality was achieved to give the product $\mathbf{4}$ using $\mathrm{HCl}$ without affecting the tosyl group. The selective deprotec-

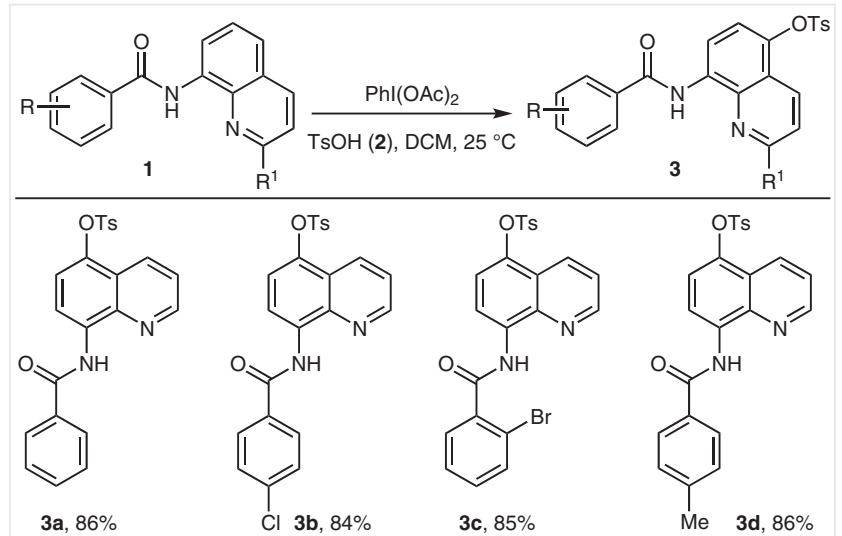<smiles>O=C(Nc1ccc(OS)c2cccnc12)c1cccc(Cl)c1</smiles><smiles>COc1ccc(NC(=O)c2cc(Br)ccc2F)c2ncccc12</smiles><smiles>O=C(Nc1ccc(O)c2cccnc12)c1ccc2ccccc2c1</smiles><smiles>COc1ccc(NC(=O)c2ccc(C(F)(F)F)cc2)c2ncccc12</smiles>

3e, $84 \%$

3f, $76 \%$<smiles>COc1ccc(NC(=O)c2cc(C(F)(F)F)cc(C(F)(F)F)c2)c2ncccc12</smiles><smiles>COc1ccc(NC(=O)c2ccc(F)cc2)c2ncccc12</smiles><smiles>CCCCC(C)(C)c1ccc(NC(=O)c2cccs2)c2ncccc12</smiles>

3i, $72 \%$

3j, $82 \%$

$3 \mathbf{k}, 81 \%$<smiles>O=C(Nc1ccc(O)c2cccnc12)c1ccc(-c2ccccc2)cc1</smiles><smiles>COc1ccc(NC(=O)c2cccc(Br)c2)c2ncccc12</smiles><smiles>COc1ccc(NC(=S)c2ccccc2)c2nc(C)ccc12</smiles>

$3 n, 89 \%$<smiles>O=C(Nc1ccc(O[Na])c2ccc(CO)nc12)c1ccccc1</smiles>

3o, $80 \%$<smiles>COc1ccc(NC(=O)c2ccc([N+](=O)[O-])cc2)c2ncccc12</smiles><smiles>COc1ccc(NC(C)=O)c2ncccc12</smiles><smiles>O=C(Nc1ccc([O-])cc1)c1ccccc1</smiles>

Scheme 3 Scope of the reaction. Yield refers to pure products after chromatography.

tion of tosyl group was accomplished to produce product $\mathbf{5}$ using $\mathrm{NaOH}$ in methanol (Scheme 4). 


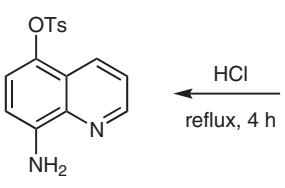<smiles>COc1ccc(NC(=O)c2ccc(C)cc2)c2ncccc12</smiles><smiles>COOCCCCCCOc1ccc(O)c2cccnc12</smiles>

Scheme 4 Deferential deprotection of amide and tosyl groups

Mechanistically, the reaction proceeds likely through a sequential formation of intermediates $\mathbf{A}$ to $\mathbf{C}$ from 8-amidoquinoline 1a and Koser's reagent. ${ }^{11}$ Finally, the displacement of aryliodonium ion by tosylate anion would give the desired product 3a (Scheme 5).<smiles>O=C(Nc1cccc2cccnc12)c1ccccc1</smiles><smiles>[O-]C(OC(=Nc1cccc2cccnc12)c1ccccc1)c1ccccc1</smiles><smiles>O=C(N=C1C=CC(B(O)c2ccccc2)c2cccnc21)c1ccccc1</smiles><smiles>O=C(N=C1C=CC([Te]c2ccccc2)c2cccnc21)c1ccccc1</smiles>

(B)

(C)<smiles>O=C(Nc1ccc(O[Na])c2cccnc12)c1ccccc1</smiles>

Scheme 5 A plausible reaction path way

In conclusion, we have developed a novel strategy for the direct oxytosylation of 8-amidoquinolines using Koser's reagent. The present strategy provides a rapid access to C-5 substituted 8-amidoquinolines in a single step process under mild conditions. It is totally a metal-free approach.

${ }^{1} \mathrm{H}$ NMR spectra were recorded at $500 \mathrm{MHz}, 300 \mathrm{MHz}$, and $400 \mathrm{MHz}$, and ${ }^{13} \mathrm{C}$ NMR at $125 \mathrm{MHz}, 100 \mathrm{MHz}$, and $75 \mathrm{MHz}$. For ${ }^{1} \mathrm{H}$ NMR, TMS was used as an internal standard $(\delta=0)$ and the values are reported as follows: chemical shift, multiplicity, integration (standard abbrevia- tions), and the coupling constants in $\mathrm{Hz}$. For ${ }^{13} \mathrm{C} \mathrm{NMR}, \mathrm{CDCl}_{3}(\delta=$ 77.00) was used as internal standard and spectra were obtained with complete proton decoupling. DMSO $(\delta=2.50)$ for ${ }^{1} \mathrm{H}$ NMR and DMSO $(\delta=39.43)$ for ${ }^{13} \mathrm{C}$ NMR. Low-resolution MS and HRMS data were obtained using ESI ionization. IR spectra were recorded on an FT-IR spectrophotometer (neat) and reported in $\mathrm{cm}^{-1}$. Melting points were measured on micro melting point apparatus. Glass syringes were used to transfer solvents. Crude products were purified by column chromatography on silica gel of $60-120$ or $100-200$ mesh. TLC plates were visualized by exposure to UV light and/or by exposure to $\mathrm{I}_{2}$ vapors and/or by exposure to methanolic acidic solution of 2-naphthol followed by heating $(<1 \mathrm{~min})$ on a hot plate $\left(250^{\circ} \mathrm{C}\right)$.

\section{$\mathbf{N}$-(Quinolin-8-yl)benzamides 1; General Procedure}

To a solution of the respective carboxylic acid $(6 \mathrm{mmol})$, the corresponding 8-aminoquinoline (6 mmol), and DMAP (73 mg, $0.6 \mathrm{mmol})$ in anhyd $\mathrm{CH}_{2} \mathrm{Cl}_{2}(30 \mathrm{~mL})$ was added a solution of EDCI $(1.38 \mathrm{~g}, 7.2$ $\mathrm{mmol})$ in $\mathrm{CH}_{2} \mathrm{Cl}_{2}(30 \mathrm{~mL})$ through a dropping funnel at $0{ }^{\circ} \mathrm{C}$ under $\mathrm{N}_{2}$ atmosphere. The mixture was allowed to stir at r.t. for overnight. After completion, the mixture was diluted with $\mathrm{CH}_{2} \mathrm{Cl}_{2}(50 \mathrm{~mL})$. The organic layer was washed with aq $1 \mathrm{~N} \mathrm{HCl}(15 \mathrm{~mL})$, followed by aq $\mathrm{NaHCO}_{3}(15$ $\mathrm{mL})$, brine $(25 \mathrm{~mL})$, and dried $\left(\mathrm{NaSO}_{4}\right)$. The organic solvent was removed by evaporation and the residue was purified by column chromatography using EtOAc/hexane to afford the desired pure $\mathrm{N}$-(quinolin-8-yl)arylamide $\mathbf{1}$.

\section{$\mathbf{N}$-(Quinolin-8-yl)benzamide (1a)}

White solid; yield: $188 \mathrm{mg}$ (94\%); $\mathrm{mp} 68-70{ }^{\circ} \mathrm{C}$.

IR (neat): 3350, 3053, 1982, 1673, 1530, 1480, 1385, 1264, 1177 , $1070,825,757,689 \mathrm{~cm}^{-1}$.

${ }^{1} \mathrm{H} \mathrm{NMR}\left(400 \mathrm{MHz}, \mathrm{CDCl}_{3}\right.$ ): $\delta=10.40$ (br s, $1 \mathrm{H}$ ), 8.93 (dd, $J=4.1,1.4$ $\mathrm{Hz}, 1 \mathrm{H}$ ), 8.25 (dd, $J=8.3,1.4 \mathrm{~Hz}, 1 \mathrm{H}), 8.10$ (d, $J=7.2 \mathrm{~Hz}, 2 \mathrm{H}$ ), 8.02 (d, $J=9.0 \mathrm{~Hz}, 1 \mathrm{H}), 7.51-7.67(\mathrm{~m}, 6 \mathrm{H})$.

${ }^{13} \mathrm{C}$ NMR $\left(\mathrm{CDCl}_{3}, 100 \mathrm{MHz}\right): \delta=165.3,148.2,138.6,136.3,135.0$, $134.5,131.8,128.8,127.9,127.2,121.7,121.6,116.5$.

HRMS (EI): $m / z(M+N a)^{+}$calcd for $\mathrm{C}_{16} \mathrm{H}_{12} \mathrm{~N}_{2} \mathrm{ONa}$ : 271.0842; found: 271.0868 .

\section{4-Chloro- $\mathrm{N}$-(quinolin-8-yl)benzamide (1b)}

Yellow solid; yield: $186 \mathrm{mg}$ (93\%); $\mathrm{mp} 100-102{ }^{\circ} \mathrm{C}$.

IR (neat): 3332, 1674, 1530, 1477, 1327, 1261, 1089, 897, 751, 662 $\mathrm{cm}^{-1}$.

${ }^{1} \mathrm{H} \mathrm{NMR}\left(500 \mathrm{MHz}, \mathrm{CDCl}_{3}\right): \delta=10.6($ br s, $1 \mathrm{H}), 8.86(\mathrm{dd}, J=7.6,1.3 \mathrm{~Hz}$, $1 \mathrm{H}), 8.78(\mathrm{dd}, J=4.2,1.6 \mathrm{~Hz}, 1 \mathrm{H}), 8.10(\mathrm{dd}, J=8.2,1.6 \mathrm{~Hz}, 1 \mathrm{H}), 7.96$ (d, $J=8.5 \mathrm{~Hz}, 2 \mathrm{H}), 7.38-7.56(\mathrm{~m}, 5 \mathrm{H})$.

${ }^{13} \mathrm{C}$ NMR $\left(\mathrm{CDCl}_{3}, 100 \mathrm{MHz}\right): \delta=164.1,148.3,138.6,138.0,136.3$, 134.3, 133.4, 129.0, 128.6, 127.9, 127.3, 121.8, 121.7, 116.5.

HRMS (EI): $m / z(M+H)^{+}$calcd for $\mathrm{C}_{16} \mathrm{H}_{12} \mathrm{ClN}_{2} \mathrm{O}$ : 283.0632; found: 283.0639.

\section{2-Bromo- $\mathbf{N}$-(quinolin-8-yl)benzamide (1c)}

Light brown solid; yield: $178 \mathrm{mg}(89 \%) ; \mathrm{mp} 86-88^{\circ} \mathrm{C}$.

IR (neat): 3334, 2924, 2853, 1673, 1529, 1480. 1420, 1324, 1259, $1065,896,792,653 \mathrm{~cm}^{-1}$.

${ }^{1} \mathrm{H}$ NMR $\left(500 \mathrm{MHz}, \mathrm{CDCl}_{3}\right.$ ): $\delta=10.66$ (br s, $1 \mathrm{H}$ ), 8.87 (dd, $J=7.4,1.3$ $\mathrm{Hz}, 1 \mathrm{H}), 8.82(\mathrm{dd}, J=4.1,1.5 \mathrm{~Hz}, 1 \mathrm{H}), 8.19(\mathrm{t}, J=1.8 \mathrm{~Hz}, 1 \mathrm{H}), 8.14$ (dd, $J=8.2,1.5 \mathrm{~Hz}, 1 \mathrm{H}), 7.95(\mathrm{~d}, J=7.7 \mathrm{~Hz}, 1 \mathrm{H}), 7.67$ (d, $J=7.9 \mathrm{~Hz}, 1 \mathrm{H})$, 7.50-7.57 (m, $2 \mathrm{H}), 7.42-7.46(\mathrm{~m}, 1 \mathrm{H}), 7.38(\mathrm{t}, J=7.9 \mathrm{~Hz}, 1 \mathrm{H})$. 
${ }^{13} \mathrm{C}$ NMR $\left(\mathrm{CDCl}_{3}, 100 \mathrm{MHz}\right): \delta=163.8,148.4,138.6,137.0,136.4$, 134.8, 134.2, 130.6, 130.3, 127.9, 127.4, 125.6, 123.0, 122.0, 121.7, 116.7.

HRMS (EI): $m / z(M)^{+}$calcd for $\mathrm{C}_{16} \mathrm{H}_{11} \mathrm{BrN}_{2} \mathrm{O}$ : 327.0128; found: 327.0150 .

\section{4-Methyl- $\mathbf{N}$-(quinolin-8-yl)benzamide (1d)}

White solid; yield: $184 \mathrm{mg}$ (92\%); mp $136-138^{\circ} \mathrm{C}$.

IR (neat): 3348, 2972, 1674, 1610, 1532, 1418, 1284, 1181, 1117, 944, $826,753,662 \mathrm{~cm}^{-1}$.

${ }^{1} \mathrm{H}$ NMR $\left(500 \mathrm{MHz}, \mathrm{CDCl}_{3}\right.$ ): $\delta=10.66$ (br s, $1 \mathrm{H}$ ), 8.98 (dd, $J=7.6,1.2$ $\mathrm{Hz}, 1 \mathrm{H}), 8.84(\mathrm{dd}, J=4.4,1.6 \mathrm{~Hz}, 1 \mathrm{H}), 8.56(\mathrm{~s}, 1 \mathrm{H}), 8.09-8.14(\mathrm{~m}, 2 \mathrm{H})$, 7.98-8.01 (m, $1 \mathrm{H}), 7.95$ (d, J = 8.5 Hz, $1 \mathrm{H}), 7.86-7.89$ (m, $1 \mathrm{H}), 7.49-$ $7.60(\mathrm{~m}, 4 \mathrm{H}), 7.41-7.44(\mathrm{~m}, 1 \mathrm{H})$.

${ }^{13} \mathrm{C}$ NMR $\left(\mathrm{CDCl}_{3}, 125 \mathrm{MHz}\right): \delta=165.4,148.2,142.3,138.8,136.3$, 134.7, 132.3, 129.4, 128.8, 128.0, 127.4, 127.3, 121.6, 121.5, 116.4, 21.5.

HRMS (EI): $m / z(M+H)^{+}$calcd for $\mathrm{C}_{17} \mathrm{H}_{15} \mathrm{~N}_{2} \mathrm{O}$ : 263.1178; found: 263.1184.

\section{3-Chloro- $\mathrm{N}$-(quinolin-8-yl)benzamide (1e)}

Yellow solid; yield: $186 \mathrm{mg}$ (93\%); $\mathrm{mp} 80-82^{\circ} \mathrm{C}$.

IR (neat): 3736, 3355, 3055, 2358, 1670, 1536, 1476, 1383, 1330, $1255,1176,904,787,762,727,678,648 \mathrm{~cm}^{-1}$.

${ }^{1} \mathrm{H} \mathrm{NMR}\left(400 \mathrm{MHz}, \mathrm{CDCl}_{3}\right): \delta=10.56$ (br s, $\left.1 \mathrm{H}\right), 8.82(\mathrm{dd}, J=7.4,1.4$ $\mathrm{Hz}, 1 \mathrm{H}), 8.73(\mathrm{dd}, J=4.2,1.7 \mathrm{~Hz}, 1 \mathrm{H}), 8.03(\mathrm{dd}, J=8.1,1.5 \mathrm{~Hz}, 1 \mathrm{H})$, $7.98(\mathrm{t}, J=1.8 \mathrm{~Hz}, 1 \mathrm{H}), 7.84(\mathrm{dt}, J=7.8,1.1 \mathrm{~Hz}, 1 \mathrm{H}), 7.32-7.50(\mathrm{~m}, 5$ $\mathrm{H})$.

${ }^{13} \mathrm{C}$ NMR $\left(\mathrm{CDCl}_{3}, 125 \mathrm{MHz}\right): \delta=163.7,148.3,138.6,136.8,136.3$, 134.9, 134.1, 131.8, 130.0, 127.9, 127.7, 127.3, 125.1, 122.0, 121.7, 116.6 .

HRMS (EI): $m / z(M+H)^{+}$calcd for $\mathrm{C}_{16} \mathrm{H}_{12} \mathrm{ClN}_{2} \mathrm{O}$ : 283.0633; found: 283.0636 .

\section{5-Bromo-2-fluoro- $\mathrm{N}$-(quinolin-8-yl)benzamide (1f)}

Brown solid; yield: $178 \mathrm{mg}$ (89\%); mp 280-282 ${ }^{\circ} \mathrm{C}$.

IR (neat): 3327, 3129, 2924, 1638, 1548, 1502, 1276, 1054, 1007, 820, $781,656 \mathrm{~cm}^{-1}$.

${ }^{1} \mathrm{H}$ NMR $\left(400 \mathrm{MHz}, \mathrm{CDCl}_{3}\right): \delta=11.06$ (br s, $1 \mathrm{H}$ ), 8.89 (dd, $J=7.0,1.4$ $\mathrm{Hz}, 1 \mathrm{H}), 8.81$ (dd, $J=4.0,1.3 \mathrm{~Hz}, 1 \mathrm{H}), 8.30(\mathrm{dd}, J=6.6,2.5 \mathrm{~Hz}, 1 \mathrm{H}$ ), $8.11(\mathrm{~d}, J=8.1 \mathrm{~Hz}, 1 \mathrm{H}), 7.47-7.49(\mathrm{~m}, 3 \mathrm{H}), 7.39-7.44(\mathrm{~m}, 1 \mathrm{H}), 7.07(\mathrm{t}$, $J=8.8 \mathrm{~Hz}, 1 \mathrm{H})$.

${ }^{13} \mathrm{C}$ NMR $\left(\mathrm{CDCl}_{3}, 125 \mathrm{MHz}\right): \delta=160.7,159.9,158.2,148.5,136.2$, $136.1,134.7,134.4,127.9,127.3,123.7,123.6,122.3,121.7,118.3$, 118.0, 117.6, 117.3.

HRMS (EI): $m / z(M+H)^{+}$calcd for $\mathrm{C}_{16} \mathrm{H}_{11}$ BrFNO: 345.0033 ; found: 345.0043.

\section{$\mathbf{N}$-(Quinolin-8-yl)-2-naphthamide (1g)}

Colorless solid; yield: $176 \mathrm{mg}(88 \%) ; \mathrm{mp} 118-120^{\circ} \mathrm{C}$.

IR (neat): 3354, 3046, 1668, 1525, 1482, 1322, 1129, 830, 771, 674 $\mathrm{cm}^{-1}$.

${ }^{1} \mathrm{H}$ NMR $\left(500 \mathrm{MHz}, \mathrm{CDCl}_{3}\right.$ ): $\delta=10.66(\mathrm{br} \mathrm{s}, 1 \mathrm{H}), 8.98(\mathrm{dd}, J=7.6,1.2$ $\mathrm{Hz}, 1 \mathrm{H}), 8.84$ (dd, $J=4.4,1.6 \mathrm{~Hz}, 1 \mathrm{H}), 8.56(\mathrm{~s}, 1 \mathrm{H}), 8.09-8.14(\mathrm{~m}, 2 \mathrm{H})$, 7.98-8.01 (m, $1 \mathrm{H}), 7.95$ (d, J = 8.5 Hz, $1 \mathrm{H}), 7.86-7.89$ (m, $1 \mathrm{H}), 7.49-$ 7.60 (m, $4 \mathrm{H}), 7.41-7.44(\mathrm{~m}, 1 \mathrm{H})$.
${ }^{13} \mathrm{C}$ NMR $\left(\mathrm{CDCl}_{3}, 100 \mathrm{MHz}\right): \delta=165.4,148.3,138.8,136.4,134.9$, 134.6, 132.7, 132.3, 129.2, 128.7, 128.0, 127.9, 127.89, 127.83, 127.5, 126.8, 123.7, 121.7.

HRMS (EI): $m / z(M+H)^{+}$calcd for $\mathrm{C}_{20} \mathrm{H}_{15} \mathrm{~N}_{2} \mathrm{O}$ : 299.1178; found: 299.1181.

\section{$\mathbf{N}$-(Quinolin-8-yl)-4-(trifluoromethyl)benzamide (1h)}

White solid; yield: $180 \mathrm{mg}$ (90\%); $\mathrm{mp} 92-94^{\circ} \mathrm{C}$.

IR (neat): 3343, 2929, 1669, 1539, 1480, 1326, 1141, 1065, 1013, 828, $600 \mathrm{~cm}^{-1}$.

${ }^{1} \mathrm{H}$ NMR $\left(500 \mathrm{MHz}, \mathrm{CDCl}_{3}\right): \delta=10.66$ (br s, $\left.1 \mathrm{H}\right), 8.84(\mathrm{~d}, J=7.4 \mathrm{~Hz}, 1$ $\mathrm{H}), 8.75(\mathrm{~d}, J=4.1 \mathrm{~Hz}, 1 \mathrm{H}), 8.07(\mathrm{t}, J=8.8 \mathrm{~Hz}, 3 \mathrm{H}), 7.70(\mathrm{~d}, J=8.0 \mathrm{~Hz}, 2$ H), 7.42-7.52 (m, $2 \mathrm{H})$, 7.34-7.39 ( $\mathrm{m}, 1 \mathrm{H})$.

${ }^{13} \mathrm{C}$ NMR $\left(\mathrm{CDCl}_{3}, 125 \mathrm{MHz}\right): \delta=163.7,148.3,138.5,138.2,136.3$, $134.0,133.2\left(\mathrm{q}, J_{\mathrm{C}, \mathrm{F}}=32.6 \mathrm{~Hz}\right), 127.8,127.6,127.2,125.7,125.7,124.8$, 122.6, 122.1, 121.7, 116.6.

HRMS (EI): $m / z(M+H)^{+}$calcd for $\mathrm{C}_{17} \mathrm{H}_{12} \mathrm{~F}_{3} \mathrm{~N}_{2} \mathrm{O}$ : 317.0896; found: 317.0884.

\section{$\mathbf{N}$-(Quinolin-8-yl)-3,5-bis(trifluoromethyl)benzamide (1i)}

Pale yellow solid; yield: $176 \mathrm{mg}(88 \%)$; mp $156-158^{\circ} \mathrm{C}$.

IR (neat): 3334, 3062, 1677, 1545, 1487, 1376, 1276, 1189, 1119, 896, $761,695 \mathrm{~cm}^{-1}$.

${ }^{1} \mathrm{H} \mathrm{NMR}\left(400 \mathrm{MHz}, \mathrm{CDCl}_{3}\right.$ ): $\delta=10.78$ (br s, $\left.1 \mathrm{H}\right), 8.84-8.88(\mathrm{~m}, 2 \mathrm{H}$ ), $8.48(\mathrm{~s}, 2 \mathrm{H}), 8.19(\mathrm{dd}, J=8.3,1.5 \mathrm{~Hz}, 1 \mathrm{H}), 8.08(\mathrm{~s}, 1 \mathrm{H}), 7.56-7.60(\mathrm{~m}$, $2 \mathrm{H}), 7.47-7.52(\mathrm{~m}, 1 \mathrm{H})$.

${ }^{13} \mathrm{C}$ NMR $\left(\mathrm{CDCl}_{3}, 125 \mathrm{MHz}\right): \delta=162.2,148.6,138.6,137.2,136.5$, 133.7, $132.4\left(\mathrm{q}, J_{\mathrm{C}, \mathrm{F}}=33.6 \mathrm{~Hz}\right), 127.9,127.5,127.3,125.32,125.30$, 125.2, 122.6, 121.9, 116.9 .

HRMS (EI): $m / z(M+H)^{+}$calcd for $\mathrm{C}_{18} \mathrm{H}_{11} \mathrm{~F}_{6} \mathrm{~N}_{2} \mathrm{O}$ : 385.0770; found: 385.0775

\section{4-Fluoro- $\mathbf{N}$-(quinolin-8-yl)benzamide (1j)}

White solid; yield: $182 \mathrm{mg}$ (91\%); $\mathrm{mp} 89-90{ }^{\circ} \mathrm{C}$.

IR (neat): 3447, 3351, 3061,1666, 1541, 1506, 1485, 1330, 1224, 1165 , $1012,822,757,649 \mathrm{~cm}^{-1}$.

${ }^{1} \mathrm{H} \mathrm{NMR}\left(500 \mathrm{MHz}, \mathrm{CDCl}_{3}\right.$ ): $\delta=10.66$ (br s, $1 \mathrm{H}$ ), 8.89 (dd, $J=7.4,1.3$ $\mathrm{Hz}, 1 \mathrm{H}), 8.82$ (dd, $J=4.1,1.6 \mathrm{~Hz}, 1 \mathrm{H}), 8.15$ (dd, $J=8.2,1.6 \mathrm{~Hz}, 1 \mathrm{H}$ ), 8.05-8.10 (m, 2 H), 7.50-7.59 (m, 2 H), 7.43-7.46 (m, 1 H), $7.20(\mathrm{t}, J=$ $8.6 \mathrm{~Hz}, 2 \mathrm{H})$.

${ }^{13} \mathrm{C}$ NMR $\left(\mathrm{CDCl}_{3}, 100 \mathrm{MHz}\right): \delta=166.2,164.2,163.7,148.3,138.7$, 136.4, 134.4, 131.3, 129.7, 129.6, 128.0, 127.4, 121.8, 121.7, 116.5, 115.9, 115.7.

HRMS (EI): $m / z(M+H)^{+}$calcd for $\mathrm{C}_{16} \mathrm{H}_{12} \mathrm{FN}_{2} \mathrm{O}$ : 267.0928; found: 267.0935 .

\section{$\mathbf{N}$-(Quinolin-8-yl)thiophene-2-carboxamide (1k)}

Pale yellow solid; yield: $150 \mathrm{mg}(75 \%) ; \mathrm{mp} 88-90{ }^{\circ} \mathrm{C}$.

IR (neat): 3346, 3073, 2924, 1651, 1533, 1483, 1355, 1267, 1115, $1055,819,731,643 \mathrm{~cm}^{-1}$.

${ }^{1} \mathrm{H} \mathrm{NMR}\left(400 \mathrm{MHz}, \mathrm{CDCl}_{3}\right): \delta=10.58$ (br s, $\left.1 \mathrm{H}\right), 8.82-8.85(\mathrm{~m}, 2 \mathrm{H})$, $8.16(\mathrm{dd}, J=8.3,1.7 \mathrm{~Hz}, 1 \mathrm{H}), 7.83(\mathrm{dd}, J=3.7,1.1 \mathrm{~Hz}, 1 \mathrm{H}), 7.50-7.59$ (m, $3 \mathrm{H}), 7.44-7.48(\mathrm{~m}, 1 \mathrm{H}), 7.16-7.19(\mathrm{~m}, 1 \mathrm{H})$.

${ }^{13} \mathrm{C}$ NMR $\left(\mathrm{CDCl}_{3}, 100 \mathrm{MHz}\right): \delta=160.0,148.3,140.1,138.5,136.3$, 134.3, 130.9, 128.4, 127.99, 127.90, 127.4, 121.75, 121.71, 116.5. 
HRMS (EI): $m / z(M+H)^{+}$calcd for $\mathrm{C}_{14} \mathrm{H}_{11} \mathrm{~N}_{2} \mathrm{OS}$ : 255.0586; found: 255.0593 .

\section{$N$-(Quinolin-8-yl)-[1,1'-biphenyl]-4-carboxamide (11)}

White solid; yield: $170 \mathrm{mg}$ (85\%); mp $139-141{ }^{\circ} \mathrm{C}$.

IR (neat): 3359, 3026, 2360, 1664, 1536, 1483, 1386, 1327, 1258, $1177,1002,895,824,743,643 \mathrm{~cm}^{-1}$.

${ }^{1} \mathrm{H} \mathrm{NMR}\left(500 \mathrm{MHz}, \mathrm{CDCl}_{3}\right): \delta=10.77$ (br s, $\left.1 \mathrm{H}\right), 8.95(\mathrm{~d}, J=7.6 \mathrm{~Hz}, 1$ $\mathrm{H}), 8.84(\mathrm{~d}, J=3.9 \mathrm{~Hz}, 1 \mathrm{H}), 8.14(\mathrm{~d}, J=8.2 \mathrm{~Hz}, 3 \mathrm{H}), 7.74(\mathrm{~d}, J=8.0 \mathrm{~Hz}$, $2 \mathrm{H}), 7.64(\mathrm{~d}, J=7.4 \mathrm{~Hz}, 2 \mathrm{H}), 7.36-7.60(\mathrm{~m}, 6 \mathrm{H})$.

${ }^{13} \mathrm{C}$ NMR $\left(\mathrm{CDCl}_{3}, 100 \mathrm{MHz}\right): \delta=165.1,148.3,144.6,140.0,138.8$, 136.4, 134.6, 133.8, 129.0, 128.1, 128.0, 127.8, 127.5, 127.4, 127.2, $121.7,116.6$

HRMS (EI): $m / z(M+H)^{+}$calcd for $\mathrm{C}_{22} \mathrm{H}_{17} \mathrm{~N}_{2} \mathrm{O}$ : 325.1335; found: 325.1366 .

\section{3-Bromo- $\mathbf{N}$-(quinolin-8-yl)benzamide (1m)}

Light brown solid; yield: $178 \mathrm{mg}$ (89\%); $\mathrm{mp} 88-90^{\circ} \mathrm{C}$.

IR (neat): 3421, 3333, 2924, 1673, 1526, 1476, 1419, 1325, 1256, $1062,901,828,733 \mathrm{~cm}^{-1}$.

${ }^{1} \mathrm{H}$ NMR $\left(500 \mathrm{MHz}, \mathrm{CDCl}_{3}\right): \delta=10.65(\mathrm{br} \mathrm{s}, 1 \mathrm{H}), 8.88(\mathrm{dd}, J=7.4,1.5$ $\mathrm{Hz}, 1 \mathrm{H}), 8.83$ (dd, $J=4.2,1.6 \mathrm{~Hz}, 1 \mathrm{H}), 8.19(\mathrm{t}, J=1.8 \mathrm{~Hz}, 1 \mathrm{H}), 8.15$ (dd, $J=8.2,1.5 \mathrm{~Hz}, 1 \mathrm{H}), 7.96(\mathrm{dt}, J=7.9,1.0 \mathrm{~Hz}, 1 \mathrm{H}), 7.68(\mathrm{~d}, J=7.9 \mathrm{~Hz}, 1$ H), 7.51-7.59 (m, $2 \mathrm{H}), 7.43-7.48(\mathrm{~m}, 1 \mathrm{H}), 7.39$ (t, $J=7.7 \mathrm{~Hz}, 1 \mathrm{H})$.

${ }^{13} \mathrm{C}$ NMR $\left(\mathrm{CDCl}_{3}, 100 \mathrm{MHz}\right): \delta=163.6,148.3,138.5,136.9,136.3$, 134.7, 134.1, 130.6, 130.2, 127.9, 127.3, 125.6, 123.0, 122.0, 121.7, 116.6 .

HRMS (EI): $m / z(M)^{+}$calcd for $\mathrm{C}_{16} \mathrm{H}_{11} \mathrm{BrN}_{2} \mathrm{O}$ : 327.0128; found: 327.0129 .

\section{$\mathrm{N}$-(2-Methylquinolin-8-yl)benzamide (1n)}

White solid; yield: $299 \mathrm{mg}$ (92\%); $\mathrm{mp} 88-90{ }^{\circ} \mathrm{C}$.

IR (neat): 3331, 3025, 1668, 1541, 1467, 833, $693 \mathrm{~cm}^{-1}$.

${ }^{1} \mathrm{H}$ NMR $\left(400 \mathrm{MHz}, \mathrm{CDCl}_{3}\right): \delta=2.77(\mathrm{~s}, 3 \mathrm{H}), 7.34(\mathrm{~d}, J=8.4 \mathrm{~Hz}, 1 \mathrm{H})$, 7.47-7.59 (m, 5 H), 8.04-8.10 (m, $3 \mathrm{H}), 8.90$ (dd, J = 7.0, 1.9 Hz, $1 \mathrm{H}$ ), $10.81(\mathrm{~s}, 1 \mathrm{H})$.

${ }^{13} \mathrm{C}$ NMR $\left(100.6 \mathrm{MHz}, \mathrm{CDCl}_{3}\right): \delta=165.3,157.2,138.1,136.5,135.3$, 133.9, 131.7, 128.8, 127.2, 126.4, 126.1, 122.4, 121.4, 116.5, 25.4.

HRMS (EI): $m / z(M+H)^{+}$calcd for $\mathrm{C}_{17} \mathrm{H}_{15} \mathrm{~N}_{2} \mathrm{O}$ : 263.1184; found: 263.1169 .

\section{$\mathrm{N}$-[2-(Hydroxymethyl)quinolin-8-yl]benzamide (10)}

Colorless solid; yield: $290 \mathrm{mg}$ (85\%); mp $116-118{ }^{\circ} \mathrm{C}$.

IR (neat): 3444, 3332, 3049, 2929, 1646, 1542, 1408, 1065, $693 \mathrm{~cm}^{-1}$.

${ }^{1} \mathrm{H}$ NMR (400 MHz, CD ${ }_{3} \mathrm{OD}$ ): $\delta=4.96(\mathrm{~s}, 2 \mathrm{H}), 7.56-7.71(\mathrm{~m}, 6 \mathrm{H}), 8.09$ (d, $J=7.4 \mathrm{~Hz}, 2 \mathrm{H}$ ), 8.34 (d, $J=8.5 \mathrm{~Hz}, 1 \mathrm{H}), 8.80$ (d, $J=7.5 \mathrm{~Hz}, 1 \mathrm{H}$ ).

${ }^{13} \mathrm{C}$ NMR (100.6 MHz, CD $\left.{ }_{3} \mathrm{OD}\right): \delta=166.0,160.0,137.8,136.9,134.7$, 133.8, 131.8, 128.6, 127.2, 126.9, 126.2, 122.0, 119.4, 116.7, 65.0.

HRMS (EI): $m / z(M+H)^{+}$calcd for $\mathrm{C}_{17} \mathrm{H}_{15} \mathrm{~N}_{2} \mathrm{O}_{2}$ : 279.1134; found: 279.1115 .

\section{4-Nitro- $N$-(quinolin-8-yl)benzamide (1p)}

Yellow solid; yield: $120 \mathrm{mg}$ (60\%); $\mathrm{mp} 154-156{ }^{\circ} \mathrm{C}$.

IR (neat): 3444, 3352, 3102, 2924, 2853, 1677, 1517, 1479, 1340, $1256,1107,824,708 \mathrm{~cm}^{-1}$.
${ }^{1} \mathrm{H}$ NMR (400 MHz, $\mathrm{CDCl}_{3}$ ): $\delta=10.79$ (br s, $1 \mathrm{H}$ ), 8.89 (dd, $J=5.9,2.9$ $\mathrm{Hz}, 1 \mathrm{H}), 8.86$ (dd, $J=4.2,1.7 \mathrm{~Hz}, 1 \mathrm{H}), 8.36(\mathrm{~d}, J=8.8 \mathrm{~Hz}, 2 \mathrm{H}), 8.20$ (d, $J=8.6 \mathrm{~Hz}, 3 \mathrm{H}), 7.58-7.61(\mathrm{~m}, 2 \mathrm{H}), 7.48-7.53(\mathrm{~m}, 1 \mathrm{H})$.

${ }^{13} \mathrm{C}$ NMR $\left(\mathrm{CDCl}_{3}, 100 \mathrm{MHz}\right): \delta=163.1,149.7,148.5,140.5,138.6$, $136.5,133.9,128.4,128.0,127.4,124.0,122.5,121.9,116.9$.

HRMS (EI): $m / z$ calcd for $\mathrm{C}_{16} \mathrm{H}_{12} \mathrm{~N}_{3} \mathrm{O}_{3}(\mathrm{M}+\mathrm{H})^{+}$: 294.0873; found: 294.0880 .

\section{$\mathbf{N}$-(Quinolin-8-yl)acetamide (1q)}

Colorless solid; yield: $158 \mathrm{mg}(79 \%) ; \mathrm{mp} 76-78{ }^{\circ} \mathrm{C}$.

IR (neat): 3286, 2924, 1665, 1533, 1481, 1422, 1377, 1320, 1257, 1092, 1004, 824, 789, $691 \mathrm{~cm}^{-1}$.

${ }^{1} \mathrm{HNMR}\left(500 \mathrm{MHz}, \mathrm{CDCl}_{3}\right): \delta=9.78(\mathrm{br} \mathrm{s}, 1 \mathrm{H}), 8.79(\mathrm{dd}, J=4.2,1.7 \mathrm{~Hz}$, $1 \mathrm{H}), 8.76$ (dd, $J=7.3,1.2 \mathrm{~Hz}, 1 \mathrm{H}), 8.14(\mathrm{dd}, J=8.1,1.5 \mathrm{~Hz}, 1 \mathrm{H}), 7.41-$ $7.55(\mathrm{~m}, 3 \mathrm{H}), 2.35(\mathrm{~s}, 3 \mathrm{H})$.

${ }^{13} \mathrm{C}$ NMR $\left(\mathrm{CDCl}_{3}, 125 \mathrm{MHz}\right): \delta=168.8,148.1,138.2,136.4,134.5$, $127.9,127.4,121.6,121.4,116.4$.

HRMS (EI): $m / z(M+N a)^{+}$calcd for $\mathrm{C}_{11} \mathrm{H}_{10} \mathrm{~N}_{2} \mathrm{ONa}$ : 209.0685; found: 209.0681 .

\section{Oxytosylation; General Procedure}

To a solution of $\mathrm{PhI}(\mathrm{OAc})_{2}(509 \mathrm{mg}, 1.58 \mathrm{mmol})$ in $\mathrm{CH}_{2} \mathrm{Cl}_{2}(10 \mathrm{~mL})$ was added $\mathrm{TsOH} \cdot \mathrm{H}_{2} \mathrm{O}$ (342 $\mathrm{mg}, 1.8 \mathrm{mmol}$ ). The resulting suspension was stirred for $15 \mathrm{~min}$ at r.t. and then a solution of 8-amidoquinoline $\mathbf{1 b}$ ( $423 \mathrm{mg}, 1.5 \mathrm{mmol}$ ) in $\mathrm{CH}_{2} \mathrm{Cl}_{2}(5 \mathrm{~mL}$ ) was added rapidly. The progress of the reaction was monitored by TLC. Upon completion, the mixture was diluted with $\mathrm{H}_{2} \mathrm{O}$ and extracted with $\mathrm{CH}_{2} \mathrm{Cl}_{2}$ and the combined organic extracts were concentrateds under reduced pressure. The resulting residue was purified by column chromatography using EtOAc/hexane to afford the pure tosyloxyamide $\mathbf{3 b}$.

\section{8-Benzamidoquinolin-5-yl 4-Methylbenzenesulfonate (3a)}

White solid; yield: $156 \mathrm{mg}$ (86\%); $\mathrm{mp} 174-176{ }^{\circ} \mathrm{C}$.

IR (neat): 3353, 2922, 1673, 1529, 1485, 1369, 1182, 1002, 859, 808, $708,662,639 \mathrm{~cm}^{-1}$.

${ }^{1} \mathrm{H}$ NMR $\left(400 \mathrm{MHz}, \mathrm{CDCl}_{3}\right): \delta=10.59$ (br s, $1 \mathrm{H}$ ), 8.86 (dd, $J=4.1,1.5$ $\mathrm{Hz}, 1 \mathrm{H}), 8.79(\mathrm{~d}, J=8.6 \mathrm{~Hz}, 1 \mathrm{H}), 8.41$ (dd, $J=8.4,1.5 \mathrm{~Hz}, 1 \mathrm{H}), 8.05$ $(\mathrm{dd}, J=8.3,1.2 \mathrm{~Hz}, 2 \mathrm{H}), 7.77(\mathrm{~d}, J=8.3 \mathrm{~Hz}, 2 \mathrm{H}), 7.52-7.61(\mathrm{~m}, 3 \mathrm{H})$, $7.50(\mathrm{dd}, J=8.4,5.1 \mathrm{~Hz}, 1 \mathrm{H}), 7.33(\mathrm{~d}, J=7.9 \mathrm{~Hz}, 2 \mathrm{H}), 7.03(\mathrm{~d}, J=8.5 \mathrm{~Hz}$, $1 \mathrm{H}), 2.46(\mathrm{~s}, 3 \mathrm{H})$.

${ }^{13} \mathrm{C}$ NMR $\left(\mathrm{CDCl}_{3}, 100 \mathrm{MHz}\right): \delta=165.5,149.0,145.8,139.6,138.9$, $134.8,133.9,132.0,131.4,130.0,128.9,128.7,127.3,123.0,122.2$, $119.9,115.3,21.7$.

HRMS (EI): $m / z(M+H)^{+}$calcd for $\mathrm{C}_{23} \mathrm{H}_{19} \mathrm{~N}_{2} \mathrm{O}_{4} \mathrm{~S}$ : 419.1060; found: 419.1095 .

\section{8-(4-Chlorobenzamido)quinolin-5-yl 4-Methylbenzenesulfonate}

(3b)

White solid; yield: $152 \mathrm{mg}$ (84\%); $\mathrm{mp} 106-108^{\circ} \mathrm{C}$.

IR (neat): 3346, 2922, 1674, 1531, 1482, 1372, 1181, 1090, 1002, 856, $754,664 \mathrm{~cm}^{-1}$.

${ }^{1} \mathrm{H}$ NMR $\left(500 \mathrm{MHz}, \mathrm{CDCl}_{3}\right): \delta=10.60(\mathrm{br} \mathrm{s}, 1 \mathrm{H}), 8.85$ (dd, $J=4.2,1.2$ $\mathrm{Hz}, 1 \mathrm{H}), 8.75(\mathrm{~d}, J=8.6 \mathrm{~Hz}, 1 \mathrm{H}), 8.39(\mathrm{dd}, J=8.5,1.2 \mathrm{~Hz}, 1 \mathrm{H}), 7.98(\mathrm{~d}$, $J=8.3 \mathrm{~Hz}, 2 \mathrm{H}), 7.77(\mathrm{~d}, J=8.2 \mathrm{~Hz}, 2 \mathrm{H}), 7.48-7.52(\mathrm{~m}, 3 \mathrm{H}), 7.32$ (d, $J=$ $8.2 \mathrm{~Hz}, 2 \mathrm{H}), 7.03(\mathrm{~d}, J=8.5 \mathrm{~Hz}, 1 \mathrm{H}), 2.45(\mathrm{~s}, 3 \mathrm{H})$. 
${ }^{13} \mathrm{C}$ NMR $\left(\mathrm{CDCl}_{3}, 125 \mathrm{MHz}\right): \delta=164.3,149.0,145.9,139.8,138.8$, 138.4, 133.6, 133.1, 132.0, 131.5, 130.0, 129.1, 128.7, 123.0, 122.3, $119.9,115.3,21.7$.

HRMS (EI): $m / z(M+H)^{+}$calcd for $\mathrm{C}_{23} \mathrm{H}_{18} \mathrm{ClN}_{2} \mathrm{O}_{4} \mathrm{~S}: 453.0670$; found: 453.0678 .

\section{8-(2-Bromobenzamido)quinolin-5-yl 4-Methylbenzenesulfonate (3c)}

White solid; yield: $153 \mathrm{mg}$ (85\%); $\mathrm{mp} 138-140{ }^{\circ} \mathrm{C}$.

IR (neat): 3334, 3070, 2921, 1664, 1531, 1371, 1170, 1047, 1002, 851, $799,668 \mathrm{~cm}^{-1}$.

${ }^{1} \mathrm{H}$ NMR $\left(500 \mathrm{MHz}, \mathrm{CDCl}_{3}\right.$ ): $\delta=10.56(\mathrm{br} \mathrm{s}, 1 \mathrm{H}), 8.84(\mathrm{dd}, J=4.1,1.6$ $\mathrm{Hz}, 1 \mathrm{H}), 8.74(\mathrm{~d}, J=8.5 \mathrm{~Hz}, 1 \mathrm{H}), 8.36(\mathrm{dd}, J=8.3,1.0 \mathrm{~Hz}, 1 \mathrm{H}), 8.16(\mathrm{~s}$, $1 \mathrm{H}), 7.93(\mathrm{~d}, J=7.6 \mathrm{~Hz}, 1 \mathrm{H}), 7.75(\mathrm{~d}, J=8.2 \mathrm{~Hz}, 2 \mathrm{H}), 7.68(\mathrm{~d}, J=7.9 \mathrm{~Hz}$, $1 \mathrm{H}), 7.50$ (dd, $J=8.4,5.1 \mathrm{~Hz}, 1 \mathrm{H}), 7.40(\mathrm{t}, J=7.7 \mathrm{~Hz}, 1 \mathrm{H}), 7.31(\mathrm{~d}, J=$ $8.0 \mathrm{~Hz}, 2 \mathrm{H}), 7.04$ (d, J = 8.5 Hz, $1 \mathrm{H}), 2.44(\mathrm{~s}, 3 \mathrm{H})$.

${ }^{13} \mathrm{C}$ NMR $\left(\mathrm{CDCl}_{3}, 100 \mathrm{MHz}\right): \delta=163.8,149.1,145.9,139.8,138.8$, 136.7, 135.0, 133.4, 132.0, 131.3, 130.6, 130.3, 130.0, 128.6, 125.6, 123.1, 122.9, 122.3, 119.8, 115.4, 21.7 .

HRMS (EI): $m / z(M)^{+}$calcd for $\mathrm{C}_{23} \mathrm{H}_{17} \mathrm{BrN}_{2} \mathrm{O}_{4} \mathrm{~S}$ : 497.0165; found: 497.0188.

\section{8-(4-Methylbenzamido)quinolin-5-yl 4-Methylbenzenesulfonate (3d)}

White solid; yield: $156 \mathrm{mg}$ (86\%); $\mathrm{mp} 122-124^{\circ} \mathrm{C}$.

IR (neat): 3362, 3926, 1671, 1530, 1468, 1370, 1184, 1002, 857, 735, $659,537 \mathrm{~cm}^{-1}$.

${ }^{1} \mathrm{H} \mathrm{NMR}\left(400 \mathrm{MHz}, \mathrm{CDCl}_{3}\right.$ ): $\delta=10.73(\mathrm{br} \mathrm{s}, 1 \mathrm{H}), 8.85(\mathrm{dd}, J=4.1,1.5$ $\mathrm{Hz}, 1 \mathrm{H}), 8.78$ (d, J = 8.6 Hz, $1 \mathrm{H}), 8.39$ (dd, $J=8.4,1.5 \mathrm{~Hz}, 1 \mathrm{H}), 7.94(\mathrm{~d}$, $J=8.1 \mathrm{~Hz}, 2 \mathrm{H}), 7.76(\mathrm{~d}, J=8.3 \mathrm{~Hz}, 2 \mathrm{H}), 7.49(\mathrm{dd}, J=8.5,4.2 \mathrm{~Hz}, 1 \mathrm{H})$, $7.33(\mathrm{t}, J=8.3 \mathrm{~Hz}, 4 \mathrm{H}), 7.02(\mathrm{~d}, J=8.5 \mathrm{~Hz}, 1 \mathrm{H}), 2.46(\mathrm{~s}, 6 \mathrm{H})$.

${ }^{13} \mathrm{C} \mathrm{NMR}\left(\mathrm{CDCl}_{3}, 100 \mathrm{MHz}\right): \delta=165.4,148.9,145.8,142.6,139.5$, 138.8, 134.0, 132.0, 131.9, 131.3, 130.0, 129.5, 128.7, 127.3, 123.0, 122.2, 119.9, 115.1, 21.7, 21.5 .

HRMS (EI): $m / z(\mathrm{M}+\mathrm{Na})^{+}$calcd for $\mathrm{C}_{24} \mathrm{H}_{20} \mathrm{~N}_{2} \mathrm{O}_{4} \mathrm{SNa}$ : 455.1605; found: 455.1620 .

\section{8-(3-Chlorobenzamido)quinolin-5-yl 4-Methylbenzenesulfonate} (3e)

White solid; yield: $152 \mathrm{mg}$ (84\%); mp $146-148^{\circ} \mathrm{C}$.

IR (neat): 3333, 3077, 2920, 2363, 1666, 1531, 1370, 1170, 1002, 851, $799,718,660 \mathrm{~cm}^{-1}$.

${ }^{1} \mathrm{H} \mathrm{NMR}\left(500 \mathrm{MHz}, \mathrm{CDCl}_{3}\right): \delta=10.59(\mathrm{br} \mathrm{s}, 1 \mathrm{H}), 8.86(\mathrm{dd}, J=4.2,1.6$ $\mathrm{Hz}, 1 \mathrm{H}), 8.75(\mathrm{~d}, J=8.5 \mathrm{~Hz}, 1 \mathrm{H}), 8.39(\mathrm{dd}, J=8.5,1.6 \mathrm{~Hz}, 1 \mathrm{H}), 8.02(\mathrm{t}$, $J=1.8 \mathrm{~Hz}, 1 \mathrm{H}), 7.90(\mathrm{~d}, J=8.0 \mathrm{~Hz}, 1 \mathrm{H}), 7.76(\mathrm{~d}, J=8.3 \mathrm{~Hz}, 2 \mathrm{H}), 7.55$ (d, $J=8.0 \mathrm{~Hz}, 1 \mathrm{H}), 7.45-7.52(\mathrm{~m}, 2 \mathrm{H}), 7.33(\mathrm{~d}, J=8.0 \mathrm{~Hz}, 2 \mathrm{H}), 7.04$ (d, $J=$ $8.5 \mathrm{~Hz}, 1 \mathrm{H}), 2.46(\mathrm{~s}, 3 \mathrm{H})$.

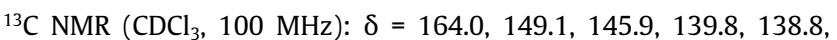
136.5, 135.1, 133.5, 132.1, 132.0, 131.4, 130.1, 130.0, 128.6, 127.7, 125.2, 123.0, 122.3, 119.9, 115.4, 21.7.

HRMS (EI): $m / z(M+H)^{+}$calcd for $\mathrm{C}_{23} \mathrm{H}_{18} \mathrm{ClN}_{2} \mathrm{O}_{4} \mathrm{~S}: 453.0670$; found: 453.0676 .

8-(2-Bromo-5-fluorobenzamido)quinolin-5-yl 4-Methylbenzenesulfonate ( $3 f$ )

Light brown solid; yield: $138 \mathrm{mg}(76 \%) ; \mathrm{mp} 120-122{ }^{\circ} \mathrm{C}$.
IR (neat): 3449, 3334, 2923, 2853, 1669, 1540, 1468, 1398, 1366, $1260,1171,1042,995,853,788,661 \mathrm{~cm}^{-1}$.

${ }^{1} \mathrm{H} \mathrm{NMR}\left(400 \mathrm{MHz}, \mathrm{CDCl}_{3}\right): \delta=10.73(\mathrm{br} \mathrm{s}, 1 \mathrm{H}), 8.86(\mathrm{dd}, J=4.1,1.3$ $\mathrm{Hz}, 1 \mathrm{H}), 8.79$ (d, $J=8.5 \mathrm{~Hz}, 1 \mathrm{H}), 8.38(\mathrm{dd}, J=8.4,1.3 \mathrm{~Hz}, 1 \mathrm{H}), 8.31$ (dd, $J=6.7,2.5 \mathrm{~Hz}, 1 \mathrm{H}), 7.77$ (d, $J=8.3 \mathrm{~Hz}, 2 \mathrm{H}), 7.61-7.66(\mathrm{~m}, 1 \mathrm{H})$, 7.49 (dd, $J=8.5,4.1 \mathrm{~Hz}, 1 \mathrm{H}), 7.33(\mathrm{~d}, J=8.0 \mathrm{~Hz}, 2 \mathrm{H}), 7.14(\mathrm{dd}, J=11.0$, $8.8 \mathrm{~Hz}, 1 \mathrm{H}), 7.06(\mathrm{~d}, J=8.5 \mathrm{~Hz}, 1 \mathrm{H}), 2.45(\mathrm{~s}, 3 \mathrm{H})$.

${ }^{13} \mathrm{C}$ NMR $\left(\mathrm{CDCl}_{3}, 100 \mathrm{MHz}\right): \delta=160.7,160.1,158.2,149.2,145.8$, 140.1, 138.9, 136.59, 136.5, 134.7, 133.8, 132.0, 131.3, 130.0, 128.6, 123.5, 123.3, 122.9, 122.3, 119.8, 118.4, 118.1, 117.7, 116.1, 21.7.

HRMS (EI): $m / z(M)^{+}$calcd for $\mathrm{C}_{23} \mathrm{H}_{16} \mathrm{BrFN}_{2} \mathrm{O}_{4} \mathrm{~S}$ : 515.0071; found: 515.0088 .

8-(2-Naphthamido)quinolin-5-yl 4-Methylbenzenesulfonate (3g) White solid; yield: $145 \mathrm{mg}$ (80\%); $\mathrm{mp} 126-128^{\circ} \mathrm{C}$.

IR (neat): 3358, 2922, 1667, 1529, 1488, 1367, 1182, 1048, 1005, 857, $730,657 \mathrm{~cm}^{-1}$.

${ }^{1} \mathrm{H}$ NMR $\left(400 \mathrm{MHz}, \mathrm{CDCl}_{3}\right.$ ): $\delta=10.73(\mathrm{br} \mathrm{s}, 1 \mathrm{H}), 8.88(\mathrm{dd}, J=4.1,1.4$ $\mathrm{Hz}, 1 \mathrm{H}), 8.84(\mathrm{~d}, J=8.5 \mathrm{~Hz}, 1 \mathrm{H}), 8.55(\mathrm{~s}, 1 \mathrm{H}), 8.40(\mathrm{dd}, J=8.5,1.5 \mathrm{~Hz}$, $1 \mathrm{H}), 7.96-8.10(\mathrm{~m}, 3 \mathrm{H}), 7.91(\mathrm{~d}, J=8.8 \mathrm{~Hz}, 1 \mathrm{H}), 7.77(\mathrm{~d}, J=8.3 \mathrm{~Hz}, 2$ H), 7.56-7.61 (m, $2 \mathrm{H}), 7.50(\mathrm{dd}, J=8.5,4.1 \mathrm{~Hz}, 1 \mathrm{H}), 7.32(\mathrm{~d}, J=8.1 \mathrm{~Hz}$, $2 \mathrm{H}), 7.06(\mathrm{~d}, J=8.6 \mathrm{~Hz}, 1 \mathrm{H}), 2.46(\mathrm{~s}, 3 \mathrm{H})$.

${ }^{13} \mathrm{C} \mathrm{NMR}\left(\mathrm{CDCl}_{3}, 125 \mathrm{MHz}\right): \delta=165.5,149.0,145.8,139.6,138.9$, 135.0, 133.9, 132.7, 132.09, 132.01, 131.4, 130.0, 129.2, 128.8, 128.7, 128.0, 127.8, 126.9, 123.6, 123.0, 122.2, 119.9, 115.3, 21.7.

HRMS (EI): $m / z(M)^{+}$calcd for $\mathrm{C}_{27} \mathrm{H}_{20} \mathrm{~N}_{2} \mathrm{O}_{4} \mathrm{~S}$ : 469.1217; found: 469.1235 .

\section{8-[4-(Trifluoromethyl)benzamido]quinolin-5-yl 4-Methylben-} zenesulfonate (3h)

White solid; yield: $126 \mathrm{mg}$ (70\%); mp 132-134 ${ }^{\circ} \mathrm{C}$.

IR (neat): 3355, 1678, 1532, 1487, 1327, 1186, 1065, 1002, 858, 737, $660 \mathrm{~cm}^{-1}$.

${ }^{1} \mathrm{H} \mathrm{NMR}\left(400 \mathrm{MHz}, \mathrm{CDCl}_{3}\right): \delta=10.66$ (br s, $\left.1 \mathrm{H}\right), 8.85$ (d, $J=3.9 \mathrm{~Hz}, 1$ $\mathrm{H}), 8.76(\mathrm{~d}, J=8.5 \mathrm{~Hz}, 1 \mathrm{H}), 8.40(\mathrm{~d}, J=8.4 \mathrm{~Hz}, 1 \mathrm{H}), 8.15(\mathrm{~d}, J=8.0 \mathrm{~Hz}$, $2 \mathrm{H}), 7.75-7.83(\mathrm{~m}, 4 \mathrm{H}), 7.50(\mathrm{dd}, J=8.4,4.1 \mathrm{~Hz}, 1 \mathrm{H}), 7.30-7.36(\mathrm{~m}, 2$ H), $7.05(\mathrm{~d}, J=8.5 \mathrm{~Hz}, 1 \mathrm{H}), 2.46(\mathrm{~s}, 3 \mathrm{H})$.

${ }^{13} \mathrm{C}$ NMR $\left(\mathrm{CDCl}_{3}, 100 \mathrm{MHz}\right): \delta=164.0,149.1,145.9,140.0,138.8$, $138.0,133.6\left(\mathrm{q}, J_{C, F}=33.6 \mathrm{~Hz}\right), 133.4,132.0,131.5,130.0,128.6,127.7$, $125.98,125.94,123.0,122.4,119.9,115.5,21.7$.

HRMS (EI): $m / z(M+H)^{+}$calcd for $\mathrm{C}_{24} \mathrm{H}_{18} \mathrm{~F}_{3} \mathrm{~N}_{2} \mathrm{O}_{4} \mathrm{~S}$ : 487.0934; found: 487.0939.

8-[3,5-Bis(trifluoromethyl)benzamido]quinolin-5-yl 4-Methylbenzenesulfonate ( $3 i$ )

White solid; yield: $130 \mathrm{mg}$ (72\%); mp $132-134^{\circ} \mathrm{C}$.

IR (neat): 3285, 3063, 2927, 1678, 1541, 1366, 1280, 1132, 1047, 852, $796,684 \mathrm{~cm}^{-1}$.

${ }^{1} \mathrm{H}$ NMR $\left(400 \mathrm{MHz}, \mathrm{CDCl}_{3}\right): \delta=10.73(\mathrm{br} \mathrm{s}, 1 \mathrm{H}), 8.86(\mathrm{dd}, J=4.1,1.3$ $\mathrm{Hz}, 1 \mathrm{H}), 8.79(\mathrm{~d}, J=8.5 \mathrm{~Hz}, 1 \mathrm{H}), 8.38(\mathrm{dd}, J=8.4,1.3 \mathrm{~Hz}, 1 \mathrm{H}), 8.31$ (dd, $J=6.7,2.5 \mathrm{~Hz}, 1 \mathrm{H}), 7.77(\mathrm{~d}, J=8.3 \mathrm{~Hz}, 2 \mathrm{H}), 7.61-7.66(\mathrm{~m}, 1 \mathrm{H})$, 7.49 (dd, $J=8.5,4.1 \mathrm{~Hz}, 1 \mathrm{H}), 7.33(\mathrm{~d}, J=8.0 \mathrm{~Hz}, 2 \mathrm{H}), 7.34(\mathrm{~d}, J=8.5 \mathrm{~Hz}$, $2 \mathrm{H}), 7.09(\mathrm{~d}, J=8.5 \mathrm{~Hz}, 1 \mathrm{H}), 2.46(\mathrm{~s}, 3 \mathrm{H})$.

${ }^{13} \mathrm{C} \mathrm{NMR}\left(\mathrm{CDCl}_{3}, 125 \mathrm{MHz}\right): \delta=162.4,149.3,145.9,140.3,138.8$, 136.9, 132.9, 132.7, 132.4, 132.1, 132.0, 131.6, 130.0, 128.6, 127.5, $125.5,124.0,123.1,122.5,121.8,119.8,115.9,21.7$. 
HRMS (EI): $m / z(M+N a)^{+}$calcd for $\mathrm{C}_{25} \mathrm{H}_{17} \mathrm{~F}_{6} \mathrm{~N}_{2} \mathrm{O}_{4} \mathrm{SNa}$ : 555.0808; found: 555.0846 .

\section{8-(4-Fluorobenzamido)quinolin-5-yl 4-Methylbenzenesulfonate}

(3j)

White solid; yield: $148 \mathrm{mg}$ (82\%); mp $182-184^{\circ} \mathrm{C}$.

IR (neat): 3349, 3072, 2923, 1674, 1598, 1532, 1485, 1372, 1328, $1233,1182,1000,854,736,662 \mathrm{~cm}^{-1}$.

${ }^{1} \mathrm{H} \mathrm{NMR}\left(400 \mathrm{MHz}, \mathrm{CDCl}_{3}\right.$ ): $\delta=10.6$ (br s, $1 \mathrm{H}$ ), 8.86 (dd, $J=4.2,1.5 \mathrm{~Hz}$, $1 \mathrm{H}), 8.76(\mathrm{~d}, J=8.5 \mathrm{~Hz}, 1 \mathrm{H}), 8.41(\mathrm{dd}, J=8.5,1.5 \mathrm{~Hz}, 1 \mathrm{H}), 8.04-8.09$ (m, $2 \mathrm{H}), 7.77$ (d, $J=8.3 \mathrm{~Hz}, 2 \mathrm{H}), 7.50(\mathrm{dd}, J=8.4,5.1 \mathrm{~Hz}, 1 \mathrm{H}), 7.33(\mathrm{~d}$, $J=8.0 \mathrm{~Hz}, 2 \mathrm{H}), 7.23(\mathrm{t}, J=8.5 \mathrm{~Hz}, 2 \mathrm{H}), 7.03(\mathrm{~d}, J=8.6 \mathrm{~Hz}, 1 \mathrm{H}), 2.45(\mathrm{~s}$, $3 \mathrm{H})$.

${ }^{13} \mathrm{C}$ NMR $\left(\mathrm{CDCl}_{3}, 125 \mathrm{MHz}\right): \delta=166.1,164.1,149.0,145.8,139.7$, 138.8, 133.7, 132.0, 131.5, 131.02, 131.00, 130.0, 129.7, 129.6, 128.7, 123.0, 122.3, 119.9, 116.0, 115.9, 115.3, 21.7.

HRMS (EI): $m / z(\mathrm{M}+\mathrm{H})^{+}$calcd for $\mathrm{C}_{23} \mathrm{H}_{18} \mathrm{FN}_{2} \mathrm{O}_{4} \mathrm{~S}$ : 437.0966; found: 437.0974.

\section{8-(Thiophene-2-carboxamido)quinolin-5-yl 4-Methylbenzenesul-} fonate (3k)

White solid; yield: $146 \mathrm{mg}$ (81\%); $\mathrm{mp} 130-132{ }^{\circ} \mathrm{C}$.

IR (neat): 3339, 3072, 2921, 1657, 1534, 1487, 1369, 1191, 1094, 994, $848,720,668 \mathrm{~cm}^{-1}$.

${ }^{1} \mathrm{H}$ NMR $\left(500 \mathrm{MHz}, \mathrm{CDCl}_{3}\right): \delta=10.48$ (br s, $1 \mathrm{H}$ ), 8.85 (dd, $J=2.8,1.3$ $\mathrm{Hz}, 1 \mathrm{H}), 8.68(\mathrm{~d}, J=8.5 \mathrm{~Hz}, 1 \mathrm{H}), 8.38(\mathrm{~d}, J=8.3 \mathrm{~Hz}, 1 \mathrm{H}), 7.81(\mathrm{dd}, J=$ $2.4,1.0 \mathrm{~Hz}, 1 \mathrm{H}), 7.75(\mathrm{~d}, J=8.2 \mathrm{~Hz}, 2 \mathrm{H}), 7.59(\mathrm{~d}, J=4.8 \mathrm{~Hz}, 1 \mathrm{H}), 7.47-$ $7.51(\mathrm{~m}, 1 \mathrm{H}), 7.32(\mathrm{~d}, J=8.0 \mathrm{~Hz}, 2 \mathrm{H}), 7.18(\mathrm{t}, J=4.2 \mathrm{~Hz}, 1 \mathrm{H}), 7.02(\mathrm{~d}$, $J=8.5 \mathrm{~Hz}, 1 \mathrm{H}), 2.45(\mathrm{~s}, 3 \mathrm{H})$.

${ }^{13} \mathrm{C}$ NMR $\left(\mathrm{CDCl}_{3}, 100 \mathrm{MHz}\right): \delta=160.0,149.0,145.8,139.6,138.6$, 133.6, 132.0, 131.4, 131.2, 130.0, 128.6, 128.3, 127.9, 123.0, 122.2, 119.9, 115.2, 21.7.

HRMS (EI): $m / z(M+H)^{+}$calcd for $\mathrm{C}_{21} \mathrm{H}_{17} \mathrm{~N}_{2} \mathrm{O}_{4} \mathrm{~S}_{2}$ : 425.0624; found: 425.0644 .

\section{8-([1,1'-Biphenyl]-4-ylcarboxamido)quinolin-5-yl 4-Methylben- zenesulfonate (31)}

White solid; yield: $136 \mathrm{mg}$ (75\%); mp $134-136^{\circ} \mathrm{C}$.

IR (neat): 3427, 3355, 2922, 2853, 1669, 1530, 1485, 1345, 1183 , $1100,997,843,789,676 \mathrm{~cm}^{-1}$.

$\left.{ }^{1} \mathrm{H} \mathrm{NMR} \mathrm{(400} \mathrm{MHz,} \mathrm{CDCl}_{3}\right): \delta=10.7$ (br s, $\left.1 \mathrm{H}\right), 8.88(\mathrm{dd}, J=4.0,1.2 \mathrm{~Hz}$, $1 \mathrm{H}), 8.81(\mathrm{~d}, J=8.5 \mathrm{~Hz}, 1 \mathrm{H}), 8.41(\mathrm{dd}, J=8.4,1.3 \mathrm{~Hz}, 1 \mathrm{H}), 8.13(\mathrm{~d}, J=$ $8.3 \mathrm{~Hz}, 2 \mathrm{H}), 7.78$ (d, J = 7.7 Hz, $4 \mathrm{H}$ ), 7.66 (d, $J=7.2 \mathrm{~Hz}, 2 \mathrm{H}), 7.46-7.54$ $(\mathrm{m}, 3 \mathrm{H}), 7.39-7.44(\mathrm{~m}, 1 \mathrm{H}), 7.33(\mathrm{~d}, J=8.0 \mathrm{~Hz}, 2 \mathrm{H}), 7.04(\mathrm{~d}, J=8.5 \mathrm{~Hz}$, $1 \mathrm{H}), 2.46(\mathrm{~s}, 3 \mathrm{H})$.

${ }^{13} \mathrm{C}$ NMR $\left(\mathrm{CDCl}_{3}, 125 \mathrm{MHz}\right): \delta=165.2,149.0,145.8,144.9,139.9$, 139.6, 138.9, 133.9, 133.4, 132.1, 131.4, 130.0, 129.0, 128.7, 128.1, $127.8,127.5,127.2,123.0,122.2,119.9,115.3,21.7$.

HRMS (EI): $m / z(M+H)^{+}$calcd for $\mathrm{C}_{29} \mathrm{H}_{23} \mathrm{~N}_{2} \mathrm{O}_{4} \mathrm{~S}$ : 495.1373; found: 495.1375.

\section{8-(3-Bromobenzamido)quinolin-5-yl 4-Methylbenzenesulfonate (3m)}

Colorless solid; yield: $153 \mathrm{mg}$ (85\%); $\mathrm{mp} 106-108^{\circ} \mathrm{C}$.
IR (neat): 3426, 3334, 3068, 2924, 2661, 2550, 1688, 1531, 1481, $1371,1306,1168,1046,940,804,744 \mathrm{~cm}^{-1}$.

${ }^{1} \mathrm{H}$ NMR (400 MHz, $\mathrm{CDCl}_{3}$ ): $\delta=10.58$ (br s, $1 \mathrm{H}$ ), 8.87 (d, $J=4.0 \mathrm{~Hz}, 1$ $\mathrm{H}), 8.74(\mathrm{~d}, J=8.5 \mathrm{~Hz}, 1 \mathrm{H}), 8.40(\mathrm{~d}, J=8.4 \mathrm{~Hz}, 1 \mathrm{H}), 8.18(\mathrm{~s}, 1$ H), $7.95(\mathrm{~d}, J=7.7 \mathrm{~Hz}, 1 \mathrm{H}), 7.76(\mathrm{~d}, J=8.1 \mathrm{~Hz}, 2 \mathrm{H}), 7.71(\mathrm{~d}, J=7.7 \mathrm{~Hz}$, $1 \mathrm{H}), 7.51(\mathrm{dd}, J=8.4,4.1 \mathrm{~Hz}, 1 \mathrm{H}), 7.42(\mathrm{t}, J=7.8 \mathrm{~Hz}, 1 \mathrm{H}), 7.33(\mathrm{~d}, J=$ $8.0 \mathrm{~Hz}, 2 \mathrm{H}), 7.04(\mathrm{~d}, J=8.5 \mathrm{~Hz}, 1 \mathrm{H}), 2.45(\mathrm{~s}, 3 \mathrm{H})$.

${ }^{13} \mathrm{C}$ NMR $\left(\mathrm{CDCl}_{3}, 100.6 \mathrm{MHz}\right): \delta=163.9,149.1,145.9,139.9,138.8$, 136.7, 136.6, 135.0, 133.4, 133.1, 132.0, 131.4, 130.6, 130.4, 130.0, 128.6, 125.6, 123.1, 123.0, 122.3, 119.8, 115.5, 21.7.

HRMS (EI): $m / z \quad(M)^{+}$calcd for $\mathrm{C}_{23} \mathrm{H}_{17} \mathrm{BrN}_{2} \mathrm{O}_{4} \mathrm{~S}$ : 497.0165; found: 497.0180.

\section{8-Benzamido-2-methylquinolin-5-yl 4-Methylbenzenesulfonate} (3n)

Colorless solid; yield: $220 \mathrm{mg}$ (89\%); $\mathrm{mp} 184-186{ }^{\circ} \mathrm{C}$.

IR (neat): 3340, 3055, 2921, 1668, 1531, 1367, 1183, 1024, 819, 631 $\mathrm{cm}^{-1}$.

${ }^{1} \mathrm{H}$ NMR $\left(400 \mathrm{MHz}, \mathrm{CDCl}_{3}\right): \delta=2.46(\mathrm{~s}, 3 \mathrm{H}), 2.76(\mathrm{~s}, 3 \mathrm{H}), 6.92(\mathrm{~d}, J=$ $8.5 \mathrm{~Hz}, 1 \mathrm{H}), 7.32$ (d, $J=8.6 \mathrm{~Hz}, 2 \mathrm{H}), 7.37$ (d, $J=8.6 \mathrm{~Hz}, 1 \mathrm{H}), 7.52-7.63$ $(\mathrm{m}, 3 \mathrm{H}), 7.76(\mathrm{~d}, J=8.3 \mathrm{~Hz}, 2 \mathrm{H}), 8.04(\mathrm{~d}, J=6.6 \mathrm{~Hz}, 2 \mathrm{H}), 8.29(\mathrm{~d}, J=8.5$ $\mathrm{Hz}, 1 \mathrm{H}), 8.74(\mathrm{~d}, J=8.5 \mathrm{~Hz}, 1 \mathrm{H}), 10.74(\mathrm{~s}, 1 \mathrm{H})$.

${ }^{13} \mathrm{C}$ NMR $\left(100.6 \mathrm{MHz}, \mathrm{CDCl}_{3}\right): \delta=165.4,158.2,145.8,139.8,138.4$, 134.9, 133.2, 132.08, 132.02, 131.48, 129.98, 128.92, 128.7, 127.2, 123.1, 121.1, 118.8, 115.2, 25.3, 21.8 .

HRMS (EI): $m / z(M+H)^{+}$calcd for $\mathrm{C}_{24} \mathrm{H}_{21} \mathrm{~N}_{2} \mathrm{O}_{4} \mathrm{~S}: 433.1222$; found: 433.1187.

\section{8-Benzamido-2-(hydroxymethyl)quinolin-5-yl 4-Methylbenzene-} sulfonate (3o)

Viscous liquid; yield: $192 \mathrm{mg}$ (80\%).

IR (neat): 2923, 2853, 1742, 1460, 1376, $720 \mathrm{~cm}^{-1}$.

${ }^{1} \mathrm{H} \mathrm{NMR}\left(400 \mathrm{MHz}, \mathrm{CDCl}_{3}\right): \delta=2.47(\mathrm{~s}, 3 \mathrm{H}), 5.00(\mathrm{~s}, 2 \mathrm{H}), 7.00(\mathrm{~d}, J=$ $8.6 \mathrm{~Hz}, 1 \mathrm{H}), 7.34(\mathrm{~d}, J=7.9 \mathrm{~Hz}, 2 \mathrm{H}), 7.51-7.58(\mathrm{~m}, 4 \mathrm{H}), 7.77$ (d, $J=8.4$ $\mathrm{Hz}, 2 \mathrm{H}), 8.00(\mathrm{~d}, J=8.3 \mathrm{~Hz}, 2 \mathrm{H}), 8.43(\mathrm{~d}, J=8.6 \mathrm{~Hz}, 1 \mathrm{H}), 8.80(\mathrm{~d}, J=8.6$ $\mathrm{Hz}, 1 \mathrm{H}), 10.39$ (s, $1 \mathrm{H})$.

${ }^{13} \mathrm{C}$ NMR $\left(100.6 \mathrm{MHz}, \mathrm{CDCl}_{3}\right): \delta=165.4,159.2,145.9,134.8,133.2$, $132.5,132.2,130.0,129.0,128.7,127.3,127.1,122.3,119.8,119.6$, 116.1, 65.1, 21.8.

HRMS (EI): $m / z(M+H)^{+}$calcd for $\mathrm{C}_{24} \mathrm{H}_{21} \mathrm{~N}_{2} \mathrm{O}_{5} \mathrm{~S}$ : 449.1171; found: 449.1145.

\section{Gram-Scale Synthesis of 8-(4-Methylbenzamido)quinolin-5-yl 4- Methylbenzenesulfonate (3d)}

To a solution of $\mathrm{PhI}(\mathrm{OAc})_{2}$ (1.54 g, $4.78 \mathrm{mmol}, 1.05$ equiv) in $\mathrm{CH}_{2} \mathrm{Cl}_{2}$ $(10 \mathrm{~mL})$ was added $\mathrm{TsOH} \cdot \mathrm{H}_{2} \mathrm{O}(1.04 \mathrm{mg}, 5.4 \mathrm{mmol}, 1.2$ equiv) and the resulting suspension was stirred for 5 min at r.t. Then, a solution of 4methyl- $N$-(quinolin-8-yl)benzamide (1d; $1.2 \mathrm{~g}, 4.58 \mathrm{mmol}, 1$ equiv) in $\mathrm{CH}_{2} \mathrm{Cl}_{2}(20 \mathrm{~mL})$ was added rapidly to the above suspension. The progress of the reaction was monitored by TLC. Upon completion, the mixture was diluted with $\mathrm{CH}_{2} \mathrm{Cl}_{2}(30 \mathrm{~mL})$ and $\mathrm{H}_{2} \mathrm{O}(20 \mathrm{~mL})$, and the aqueous layer was extracted with $\mathrm{CH}_{2} \mathrm{Cl}_{2}(3 \times 20 \mathrm{~mL})$. The combined organic layers were dried $\left(\mathrm{Na}_{2} \mathrm{SO}_{4}\right)$. The solvent was removed by evaporation and the residue was purified by flash chromatography to give the product 3d as a white solid; yield: $1.6 \mathrm{~g}(3.7 \mathrm{mmol}, 81 \%)$. 


\section{Deprotection of the Amide Functionality; 8-Aminoquinolin-5-yl} 4-Methylbenzenesulfonate (4)

A solution of 8-(4-methylbenzamido)quinolin-5-yl 4-methylbenzenesulfonate (3d; $500 \mathrm{mg}, 1.16 \mathrm{mmol})$ in aq $2 \mathrm{M} \mathrm{HCl}(20 \mathrm{~mL})$ was heated under reflux for $3 \mathrm{~h}$. The progress of the reaction was monitored by TLC. After complete conversion, the mixture was quenched with aq $\mathrm{NaHCO}_{3}$ and diluted with $\mathrm{CH}_{2} \mathrm{Cl}_{2}(30 \mathrm{~mL})$. The organic layer was washed with $\mathrm{H}_{2} \mathrm{O}(20 \mathrm{~mL})$ and the aqueous layer was extracted with $\mathrm{CH}_{2} \mathrm{Cl}_{2}(3 \times 20 \mathrm{~mL})$. The combined organic layers were dried $\left(\mathrm{Na}_{2} \mathrm{SO}_{4}\right)$. Removal of the solvent followed by purification on silica gel afforded the product 4 as a brown solid; yield: $90 \mathrm{mg}(80 \%)$; $\mathrm{mp} \mathrm{118-}$ $120^{\circ} \mathrm{C}$.

IR (neat): 3488, 3385, 2923, 1592, 1478, 1357, 1171, 1044, 849, 789, $660 \mathrm{~cm}^{-1}$.

${ }^{1} \mathrm{H}$ NMR $\left(400 \mathrm{MHz}, \mathrm{CDCl}_{3}\right): \delta=8.72(\mathrm{dd}, J=4.1,1.4 \mathrm{~Hz}, 1 \mathrm{H}), 8.15(\mathrm{dd}$, $J=8.5,1.5 \mathrm{~Hz}, 1 \mathrm{H}), 7.73(\mathrm{~d}, J=8.3 \mathrm{~Hz}, 1 \mathrm{H}), 7.33(\mathrm{dd}, J=8.5,4.1 \mathrm{~Hz}, 1$ $\mathrm{H}), 7.27(\mathrm{~d}, J=7.9 \mathrm{~Hz}, 2 \mathrm{H}), 6.95(\mathrm{~d}, J=8.3 \mathrm{~Hz}, 1 \mathrm{H}), 6.70(\mathrm{~d}, J=8.3 \mathrm{~Hz}$, $1 \mathrm{H}), 2.42(\mathrm{~s}, 3 \mathrm{H})$.

${ }^{13} \mathrm{C}$ NMR $\left(\mathrm{CDCl}_{3}, 125 \mathrm{MHz}\right): \delta=147.9,145.4,143.3,137.8,135.3$, $132.4,130.5,129.7,128.6,123.3,121.8,120.4,107.8,21.7$.

MS (ESI): $m / z=314(\mathrm{M}+\mathrm{Na})^{+}$.

\section{Deprotection of the Tosyl Group; $N$-(5-Hydroxyquinolin-8-yl)-4- methylbenzamide (5)}

To a stirred solution of 8-(4-methylbenzamido)quinolin-5-yl 4-methylbenzenesulfonate (3d; $500 \mathrm{mg}, 1.16 \mathrm{mmol}$ ) in anhyd MeOH $(15 \mathrm{~mL})$ was added $\mathrm{NaOH}$ ( $51 \mathrm{mg}, 1.3 \mathrm{mmol}$ ) and heated to reflux for $1 \mathrm{~h}$. The progress of the reaction was monitored by TLC. Upon completion, $\mathrm{MeOH}$ was removed under reduced pressure. The mixture was diluted with $\mathrm{CH}_{2} \mathrm{Cl}_{2}(30 \mathrm{~mL})$. The organic layer was washed with $\mathrm{H}_{2} \mathrm{O}(20 \mathrm{~mL})$ and the aqueous layer was extracted with $\mathrm{CH}_{2} \mathrm{Cl}_{2}(3 \times 20 \mathrm{~mL})$. The combined organic layers were dried $\left(\mathrm{Na}_{2} \mathrm{SO}_{4}\right)$ The solvent was removed by evaporation and the residue was purified by flash chromatography to give the product 5 as a white solid; yield: $100 \mathrm{mg}(84 \%)$; $\mathrm{mp} 214-216^{\circ} \mathrm{C}$.

IR (neat): 3328, 1644, 1548, 1503, 1283, 1189, 1010, 740, $664 \mathrm{~cm}^{-1}$.

${ }^{1} \mathrm{H} \mathrm{NMR}\left(500 \mathrm{MHz}, \mathrm{CDCl}_{3}\right): \delta=9.19(\mathrm{br} \mathrm{s}, 1 \mathrm{H}), 9.14(\mathrm{~s}, 1 \mathrm{H}), 7.67$ (d, $J=$ $3.0 \mathrm{~Hz}, 1 \mathrm{H}$ ), 7.36 (d, $J=8.2 \mathrm{~Hz}, 2 \mathrm{H}), 6.67$ (d, $J=7.9 \mathrm{~Hz}, 2 \mathrm{H}), 6.33$ (dd, $J=8.2,4.1 \mathrm{~Hz}, 1 \mathrm{H}), 6.14(\mathrm{~d}, J=7.7 \mathrm{~Hz}, 2 \mathrm{H}), 5.74(\mathrm{~d}, J=8.2 \mathrm{~Hz}, 1 \mathrm{H})$, $1.20(\mathrm{~s}, 3 \mathrm{H})$

${ }^{13} \mathrm{C}$ NMR $\left(\mathrm{CDCl}_{3}, 125 \mathrm{MHz}\right): \delta=162.4,147.2,147.0,140.1,137.5$, 130.3, 129.8, 127.6, 125.1, 124.5, 118.9, 118.0, 115.8, 106.3, 19.4.

HRMS (EI): $m / z(M+H)^{+}$calcd for $\mathrm{C}_{17} \mathrm{H}_{15} \mathrm{~N}_{2} \mathrm{O}_{2}$ : 279.1128; found: 279.1161 .

\section{Acknowledgment}

Z.B. thanks CSIR, New Delhi for the award of a fellowship.

\section{Supporting Information}

Supporting information for this article is available online at https://doi.org/10.1055/s-0037-1610212. Copies of ${ }^{1} \mathrm{H}$ and ${ }^{13} \mathrm{C}$ NMR spectra of products are provided.

\section{References}

(1) (a) Arnold, R.; Nutter, W.; Stepp, W. J. Org. Chem. 1959, 24, 117. (b) Clawson, R. W.; Deavers, R. E.; Akhmedov, N. G.; Soderberg, B. C. G. Tetrahedron 2006, 62, 10829. (c) Pan, L.; Wang, L.; Chen, Q.; He, M. Synth. Commun. 2016, 24, 198.

(2) John, O. R. S.; Killeen, N. M.; Knowles, D. A.; Yau, S. C.; Bagley, M. C.; Tomkinson, N. C. O. Org. Lett. 2007, 9, 4009.

(3) (a) Yan, Q.; Chen, Z.; Yu, W.; Yin, H.; Liu, Z.; Zhang, Y. Org. Lett. 2015, 17, 2482. (b) Ye, X.; Petersen, J. L.; Shi, X. Chem. Commun. 2015, 51, 7863. (c) Reddy, V. P.; Qiu, R.; Iwasaki, T.; Kambe, N. Org. Biomol. Chem. 2015, 13, 6803. (d) Liu, J.; Zhuang, S.; Gui, Q.; Chen, X.; Wang, W.; Ten, Z. Chem. Commun. 2015, 51, 6418. (e) Shang, R.; Ilies, L.; Nakamura, E. J. Am. Chem. Soc. 2015, 137, 7660. (f) Whiteoak, C. J.; Planas, O.; Company, A.; Ribas, X. Adv. Synth. Catal. 2016, 358, 1679. (g) Dou, Y.; Xie, Z.; Sun, Z.; Fang, H.; Shen, C.; Zhang, P. ChemCatChem 2016, 8, 3570. (h) Liang, S.; Manolikakes, G. Adv. Synth. Catal. 2016, 358, 2371. (i) Chen, H.; Li, P.; Wang, M.; Wang, L. Org. Lett. 2016, 18, 4794. (j) Arockiam, P. A.; Guillemard, L.; Delord, J. W. Adv. Synth. Catal. 2017, 359, 2571. (k) Li, J. M.; Wang, Y. H.; Yu, Y.; Wu, R. B.; Weng, J.; Lu, G. ACS Catal. 2017, 7, 2661. (l) Ghosh, T.; Maity, P.; Ranu, B. C. Org. Lett. 2018, 20, 1011. (m) Mondal, S.; Hajra, A. Org. Biomol. Chem. 2018, 16, 2846.

(4) (a) Liang, H.; Jiang, K.; Ding, W.; Yuan, Y.; Shuai, L.; Chen, Y.; Wei, Y. Chem. Commun. 2015, 51, 16928. (b) Xu, J.; Zhu, X.; Zhou, G.; Ying, B.; Ye, P.; Su, L.; Shen, C.; Zhang, P. Org. Biomol. Chem. 2016, 14, 3016. (c) Cong, X.; Zeng, X. Org. Lett. 2014, 16, 3716. (d) Liu, X.; Wu, Z.; Luo, X.; He, Y.; Zhou, X.; Fan, Y.; Huang, G. RSC Adv. 2016, 6, 71485 .

(5) Koser, G. F.; Relenyi, A. G.; Kalos, A. N.; Rebrovic, L.; Wettach, R. H. J. Org. Chem. 1982, 47, 2487.

(6) Shen, C.; Yang, M.; Xu, J.; Chen, C.; Zheng, K.; Shen, J.; Zhang, P. RSC Adv. 2017, 7, 49436.

(7) (a) Reddy, B. V. S.; Reddy, C. R.; Reddy, M. R.; Yarlagadda, S.; Sridhar, B. Org. Lett. 2015, 17, 3730. (b) Reddy, C. R.; Yarlagadda, S.; Ramesh, B.; Reddy, M. R.; Sridhar, B.; Reddy, B. V. S. Eur. J. Org. Chem. 2017, 2332.

(8) (a) Hao, X.; Chen, L.; Ren, B.; Li, L.; Yang, X.; Gong, J.; Niu, J.; Song, M. Org. Lett. 2014, 16, 1104. (b) Yang, Y.; Shi, L.; Zhou, Y.; Li, H.; Zhu, W.; Zhu, H. Bioorg. Med. Chem. 2010, 20, 6653.

(9) (a) Yusubov, M. S.; Wirth, T. Org. Lett. 2005, 7, 519. (b) Nabana, T.; Togo, H. J. Org. Chem. 2002, 67, 4362. (c) Abe, S.; Sakuratani, K.; Togo, H. J. Org. Chem. 2001, 66, 6174. (d) Tuncay, A.; Dustman, J. A.; Fisher, G.; Tuncay, C. I. Tetrahedron Lett. 1992, 33, 7647.

(10) CCDC 1553346 contains supplementary crystallographic data for the structure 3a. The data can be obtained free of charge from The Cambridge Crystallographic Data Centre via www.ccdc.cam.ac.uk/getstructures.

(11) (a) Basdevant, B.; Legault, C. Y. Org. Lett. 2015, 17, 4918. (b) Carreras, V.; Sandtorv, A. H.; Stuart, D. R. J. Org. Chem. 2017, 82,1279 . 